\title{
BANACH SPACES WITH SEPARABLE DUALS
}

\author{
M. ZIPPIN
}

\begin{abstract}
It is proved that every Banach space with a separable dual embeds into a space with a shrinking basis. It follows that every separable reflexive space can be embedded in a reflexive space with a basis.
\end{abstract}

1. Introduction. A basis $\left\{x_{n}\right\}_{n=1}^{\infty}$ of a Banach space $X$ is called shrinking if its biorthogonal functionals $\left\{x_{n}^{*}\right\}_{n=1}^{\infty}$ span the whole dual space $X^{*}$. The basis is called boundedly complete if, for every sequence of numbers $\left\{a_{n}\right\}_{n=1}^{\infty}, \sum_{n=1}^{\infty} a_{n} x_{n}$ converges whenever $\sup _{n}\left\|\sum_{i=1}^{n} a_{i} x_{i}\right\|<\infty$. It is easy to see that if $\left\{x_{n}\right\}_{n=1}^{\infty}$ is a shrinking basis of $X$, then $\left\{x_{n}^{*}\right\}_{n=1}^{\infty}$ is a boundedly complete basis of $X^{*}$. Conversely, if $\left\{x_{n}\right\}_{n=1}^{\infty}$ is a boundedly complete basis of $X$, then $\left\{x_{n}^{*}\right\}_{n=1}^{\infty}$ is a shrinking basis of its closed linear span.

The relations between spaces with separable duals and spaces with shrinking bases, on the one hand, and the relations between spaces which are themselves separable duals and spaces with boundedly complete bases, on the other hand, have been extensively studied in the literature starting with the investigations of R. C. James in the early 1950s. For example, it is known (see [4]) that if $X^{*}$ has a basis, then $X$ has a shrinking basis (the biorthogonal functions of which form a boundedly complete basis of $X^{*}$ ). In the same paper it is proved that $X$ has a shrinking basis if $X$ has a basis and $X^{*}$ is separable and has the bounded approximation property. It was proved in [2] that every space with a separable dual contains a subspace with a shrinking basis and, a year later, in [3], that every separable dual contains a subspace with a boundedly complete basis. The next important step was done in [1] where it was proved that every Banach space with a separable dual is a quotient space of a space with a shrinking basis and, hence, every separable dual space embeds into a space with a boundedly complete basis. The purpose of this paper is to prove the following

THEOREM. Let $E$ be a Banach space with a separable dual $E^{*}$. Then $E$ is isomorphic to a subspace of a Banach space with a shrinking basis.

The Theorem answers Problem 1.b.16 of [6] and, as explained in [1, p. 320], it implies the following

COROLlaRY. Let $X$ be a separable reflexive space. Then $X$ is isomorphic to a subspace of a reflexive space with a basis.

The Corollary affirmatively settles Problem 1(A1) of [7].

ACKNOWLEDGEMENT. Part of this work was done while the author was visiting Texas A \& M University. The author wishes to thank the Mathematics Department

Received by the editors September 15, 1987.

1980 Mathematics Subject Classification (1985 Revision). Primary 46B10, 46B15. 
for making this visit possible. Special thanks are due to Bill Johnson, a perfect host whose good advice helped simplify the proof considerably.

NOTATION AND DEFINITIONS. Let $\left\{y_{n}\right\}_{n=1}^{\infty}$ be a sequence of elements of a Banach space $Y$. The norm closed linear span of $\left\{y_{n}\right\}_{n=1}^{\infty}$ is denoted by $\left[y_{n}\right]_{n=1}^{\infty}$. If $\left\{y_{n}\right\}_{n=1}^{\infty}$ is a basis of $Y$, then the basis projections $\left\{P_{n}\right\}_{n=1}^{\infty}$ are defined by

$$
P_{n}\left(\sum_{i=1}^{\infty} a_{i} x_{i}\right)=\sum_{i=1}^{n} a_{i} x_{i} .
$$

The basis is called monotone if $\left\|P_{n}\right\|=1$ for all $n$. A Banach space $Y$ is said to have a finite-dimensional decomposition (f.d.d.) if there exists a sequence of commuting projections $P_{n}$ of finite rank on $Y$ such that $\sup _{n}\left\|P_{n}\right\|<\infty, P_{1}(Y) \subset P_{2}(Y) \subset \cdots$ and $\lim _{n} P_{n} y=y$ for every $y \in Y$.

Let $M$ be a positive number. A subset $A \subset X^{*}$ is called an $M$-norming set for a subspace $E \subset X$ if for every $x \in E$

$$
M^{-1} \sup \left\{x^{*}(x): x^{*} \in A\right\} \leq\|x\| \leq M \sup \left\{x^{*}(x): x^{*} \in A\right\} .
$$

$A$ is called a norming set for $E$ if it is $M$-norming for $E$ for some $M>0$. For any Banach space $X, B(X)$ denotes the closed unit ball of $X$.

THE MAIN IDEAS. The long proof of the Theorem justifies a short description of the main points. The main part of the proof is the embedding of $E$ into a space $X$ with a basis $\left\{x_{n}\right\}_{n=1}^{\infty}$ in such a way that $\left[x_{n}^{*}\right]_{n=1}^{\infty}$ contains a $\omega^{*}$-compact norming set $\Gamma$ for $E$. Lemma 1 states that, under these circumstances, it can be assumed that $\Gamma$ is a norming set for the whole space $X$. A result of Haydon [5] shows that $\Gamma$ may be assumed to be convex and a standard separation argument then implies that $\left[x_{n}^{*}\right]_{n=1}^{\infty}=X^{*}$, concluding the proof of the Theorem.

REMARK 1 . The use of Lemma 1 can be replaced by the interpolation method of $[\mathbf{1}]$. However, this interpolation argument is valid only if the set $\Gamma$ is convex. So, one may first use Haydon's result to get a convex $\omega^{*}$-compact norming set for $E$ in $\left[x_{n}^{*}\right]_{n=1}^{\infty}$ and then use the interpolation method of [1]. We believe that Lemma 1 is of some interest in its own right since it may be a useful tool replacing the interpolation method when one starts with a nonconvex set, without being allowed to convexify it, and has to stick to the smallest possible norming set.

Dealing with the problem of getting the desired set $\left(\mathrm{a} \omega^{*}\right.$-compact norming set for $E$ in the closed linear span of the biorthogonal functionals of some basis of a space $X$ containing $E$ ) we face the following difficulties: in order to be norming the set needs to be quite large. On the other hand, in order to be $\omega^{*}$-compact and containable in $\left[x_{n}^{*}\right]_{n=1}^{\infty}$ it has to be small enough. We need some concrete structure on the set $\Gamma$ in order to make the necessary computations. This is where we use what we call a Cantor partition. Let $\Delta$ be a compact metric space. A collection of subsets of $\Delta,\left\{\Delta\left(a_{1}, a_{2}, \ldots, a_{n}\right): n \geq 1, a_{i} \in D_{i}, 1 \leq i \leq n\right\}$, where $D_{1}, D_{2}, \ldots$ are finite sets of indices, is called a Cantor partition if the following four conditions are satisfied:

(1.1) each subset $\Delta\left(a_{1}, a_{2}, \ldots, a_{n}\right)$ is both closed and open (clopen, in short);

(1.2) $\Delta=\bigcup_{a_{1} \in D_{1}} \Delta\left(a_{1}\right)$ and $\Delta\left(a_{1}\right) \cap \Delta\left(a_{1}^{\prime}\right)=\varnothing$ if $a_{1} \neq a_{1}^{\prime}$;

(1.3) for every $n$ and $a_{1}, a_{2}, \ldots, a_{n}$ with $a_{i} \in D_{i}$,

$$
\Delta\left(a_{1}, a_{2}, \ldots, a_{n}\right)=\bigcup_{a_{n+1} \in D_{n+1}} \Delta\left(a_{1}, a_{2}, \ldots, a_{n}, a_{n+1}\right)
$$


and

$$
\Delta\left(a_{1}, a_{2}, \ldots, a_{n}, a_{n+1}\right) \cap \Delta\left(a_{1}, a_{2}, \ldots, a_{n}, a_{n+1}^{\prime}\right)=\varnothing \quad \text { if } a_{n+1} \neq a_{n+1}^{\prime} ;
$$

(1.4) if $a_{1}, a_{2}, \ldots$ is a sequence of indices with $a_{i} \in D_{i}$ such that, for every $n \geq 1, \Delta\left(a_{1}, a_{2}, \ldots, a_{n}\right) \neq \varnothing$, then $\bigcap_{n=1}^{\infty} \Delta\left(a_{1}, a_{2}, \ldots, a_{n}\right)$ is a singleton set $\{\delta\}$; this element $\delta$ will be denoted by $\delta\left(a_{1}, a_{2}, \ldots\right)$.

It clearly follows that $\lim _{n}$ diameter $\Delta\left(a_{1}, a_{2}, \ldots, a_{n}\right)=0$ and that every $\delta \in$ $\Delta$ is a $\delta\left(a_{1}, a_{2}, \ldots\right)$ for one sequence $a_{1}, a_{2}, \ldots$ with $a_{i} \in D_{i}$. Note that some sets $\Delta\left(a_{1}, a_{2}, \ldots, a_{n}\right)$ may be empty or singletons unlike the classical partition of Cantor's discontinuum. Given a Cantor partition of $\Delta$, a collection of points $\left\{\delta\left(a_{1}, a_{2}, \ldots, a_{n}\right): n \geq 1, a_{i} \in D_{i}\right.$ for $\left.1 \leq i \leq n\right\}$ is called a tree for the partition if $\delta\left(a_{1}, a_{2}, \ldots, a_{n}\right) \in \Delta\left(a_{1}, a_{2}, \ldots, a_{n}\right)$ for every $n$ and $a_{i} \in D_{i}, 1 \leq i \leq n$. The points $\delta\left(a_{1}, a_{2}, \ldots, a_{n}\right)$ will be called branch points of the tree. If $\delta \in \Delta$ and $\delta=\delta\left(a_{1}, a_{2}, \ldots\right)$ with $a_{i} \in D_{i}$ then $\delta=\lim _{n} \delta\left(a_{1}, a_{2}, \ldots, a_{n}\right)$.

In Lemma 3 we will construct for $E$ a $\omega^{*}$-compact norming set $\Delta$ in $E^{*}$ which, under the $\omega^{*}$-topology, has a Cantor partition with a tree having a rather special property: for every $\delta \in \Delta$, if $\delta=\delta\left(a_{1}, a_{2}, \ldots\right)$, then the branch points $\delta\left(a_{1}, a_{2}, \ldots, a_{n}\right)$ converge to $\delta$ not only in the original $\omega^{*}$ topology of $\Delta$ but also in the norm topology of $E^{*}$. We then embed $E$ into a space with a basis and embed $\Delta$ in the dual space by a $\omega^{*}-\omega^{*}$ continuous map which extends functionals; the above property of the branch points ensures that we get the desired norming set for $E$ in the closed linear span of the biorthogonal functionals.

\section{Preliminary lemmas.}

LEMMA 1. Let $E$ be a subspace of a Banach space $X$ with a basis $\left\{x_{n}\right\}_{n=1}^{\infty}$, let $\left\{x_{n}^{*}\right\}_{n=1}^{\infty}$ denote the corresponding biorthogonal functionals, and assume, that for some $M>0, B\left(\left[x_{n}^{*}\right]_{n=1}^{\infty}\right)$ contains a $\omega^{*}$-compact subset $\Omega$ which is $M$-norming for $E$. Then $E$ embeds into a Banach space $X_{1}$ with a basis $\left\{\tilde{x}_{n}\right\}_{n=1}^{\infty}$ such that $\left[\tilde{x}_{n}^{*}\right]_{n=1}^{\infty}$ contains a $\omega^{*}$-compact norming set for the whole space $X_{1}$.

PROOF. Let $Q_{n}$ denote the basis $n$th projection defined by $Q_{n}\left(\sum_{i=1}^{\infty} a_{i} x_{i}\right)=$ $\sum_{i=1}^{n} a_{i} x_{i}$. We may assume without loss of generality that, for every $n,\left\|x_{n}\right\|=$ $\left\|Q_{n}\right\|=\left\|I-Q_{n}\right\|=1$ ( $X$ can be equivalently renormed to have this property). Define the following subsets of $X^{*}$ :

$$
L=\left\{\sum_{i=1}^{\infty} a_{i} x_{i}^{*}: \sum_{i=1}^{\infty}\left|a_{i}\right| \leq 1\right\}, \quad H_{0}=\Omega \cup\{0\} \cup\left\{Q_{n} y^{*}: y^{*} \in \Omega \text { and } n \geq 1\right\}
$$

and, letting $Q_{0}^{*}=I$, the identity on $X^{*}$, we put for every $m \geq 1$

$$
\begin{array}{r}
H_{m}=\{0\} \cup\left\{\left\|\left(I-Q_{m}^{*}\right) Q_{n}^{*} y^{*}\right\|^{-1}\left(I-Q_{m}^{*}\right) Q_{n}^{*} y^{*}: y^{*} \in \Omega, n \geq 0\right. \\
\text { and } \left.\left\|\left(I-Q_{m}^{*}\right) Q_{n}^{*} y^{*}\right\|>2^{-m}\right\} .
\end{array}
$$

Finally, let $\Gamma=L \cup\left(\bigcup_{m=0}^{\infty} H_{m}\right)$ and $X_{0}=$ the linear span of $\left\{x_{n}\right\}_{n=1}^{\infty}$ and renorm $X_{0}$ by $|x|=\sup \left\{y^{*}(x): y^{*} \in \Gamma\right\}$. Let $X_{1}$ be the completion of $X_{0}$ under the norm $|\cdot|$ and denote each $x_{n}$ by $\tilde{x}_{n}$ when regarded as an element of $X_{1}$. Note that for some $n$ 's we may have $\left|\tilde{x}_{n}\right|=0$ but in this case $\tilde{x}_{n}$ may be deleted and the remaining elements $\left\{\tilde{x}_{i}:\left|\tilde{x}_{i}\right| \neq 0\right\}$ will clearly form a montone basis of $X_{1}$. For the sake of 
simpler notation we will assume that $\left|\tilde{x}_{i}\right| \neq 0$ for all $i \geq 1$. Let $G_{1}$ denote $\left[\tilde{x}_{n}^{*}\right]_{n=1}^{\infty}=$ the closed linear span of $\left\{x_{n}^{*}\right\}_{n=1}^{\infty}$ in $X_{1}^{*}$. The main point of the proof is to show that $\Omega \subset G_{1}$. Recall that each $y^{*} \in \Omega$ is, in fact, a series $y^{*}=\sum_{1}^{\infty} a_{i} y_{i}^{*}$, convergent in the original norm. If this series does not converge in the new norm then there exists a sequence $\left\{z_{n}\right\}_{n=1}^{\infty}$ of disjointly supported blocks (i.e. $z_{n}=\sum_{i=q(n)+1}^{q(n+1)} c_{i} \tilde{x}_{i}$ where $0=q(1)<q(2)<\cdots)$ such that $\left|z_{n}\right|=1$ and $\inf _{n} y^{*}\left(z_{n}\right)=d>0$. Put $w_{n}^{*}=\left(I-Q_{n}^{*}\right) y^{*}$. We claim that there is an integer $N$ such that $\left\|w_{m}^{*}\right\| \leq 2^{-m}$ for all $m \geq N$. Indeed, if this is not the case, then $\left\|w_{m}^{*}\right\|^{-1} w_{m}^{*} \in H_{m}$ infinitely often and so $1=\left|z_{m+1}\right| \geq||\left|w_{m}^{*}\left\|^{-1} w_{m}^{*}\left(z_{m+1}\right)\left|=\left\|w_{m}^{*}\right\|^{-1}\right| y^{*}\left(z_{m+1}\right) \mid \geq d\right\| w_{m}^{*} \|^{-1}\right.$, contradicting the fact that $w_{m}^{*}$ is the tail of a convergent series (in $\|\cdot\|$ ). It follows that for all $m$ large enough, $\left\|w_{m}^{*}\right\| \leq 2^{-m}$ as claimed. Since $\left\|x_{i}^{*}\right\|=1$ for all $i \geq 1$, if $i>N+1$ then

$$
\left|a_{i}\right|=\left\|a_{i} x_{i}^{*}\right\|=\left\|w_{i-1}^{*}-w_{i}^{*}\right\| \leq 2^{-(i-1)}+2^{-i} \leq 2^{-(i-2)} .
$$

Hence, if $k>N+1$, then the functional $z_{k}^{*}=2^{k} \sum_{i=k+3}^{\infty} a_{i} y_{i}^{*}$ belongs to $L$ and therefore, if $k<n$ are large enough, we get the contradiction $1=\left|z_{n}\right| \geq z_{m}^{*}\left(z_{n}\right)=$ $2^{k} y^{*}\left(z_{n}\right)=2^{k} d$. This proves that $y^{*} \in G_{1}$ and so $\Omega \subset G_{1}$. For a subset $A$ of $X_{1}^{*}$ let $\tilde{A}$ denote the $\omega^{*}$-closure of $A$. We now claim that $\tilde{\Gamma} \subset G_{1}$. Clearly $\tilde{L}=L$ and $\tilde{H}_{0} \subset G_{1}$. Let us consider the set $H_{m}$ for $m \geq 1$. Let $\left\{u_{j}^{*}\right\}_{j=1}^{\infty} \subset H_{m}$ and suppose that $\omega^{*}-\lim u_{j}^{*}=u^{*} \neq 0$. Let $u_{j}^{*}=\left\|\left(I-Q_{m}^{*}\right) Q_{n(j)}^{*}\left(z_{j}^{*}\right)\right\|^{-1}\left(I-Q_{m}^{*}\right) Q_{n(j)}^{*}\left(z_{j}^{*}\right)$ with $z_{j}^{*} \in \Omega$ be so that $\left\|\left(I-Q_{m}^{*}\right) Q_{n(j)}^{*}\left(z_{j}^{*}\right)\right\|>2^{-m}$. By passing to a subsequence we may assume that $\omega^{*}-\lim z_{j}^{*}=z^{*} \in \Omega \subset G_{1}$ and that $\{n(j)\}_{j=1}^{\infty}$ is either a constant sequence or tends to infinity. In the first case $u^{*}$ is clearly in $G_{1}$ while in the second one the sequence $Q_{n(j)}^{*}\left(z_{j}^{*}\right)-z^{*}=Q_{n(j)}^{*}\left(z_{j}^{*}-z^{*}\right)+Q_{n(j)}^{*}\left(z^{*}\right)-z^{*}$ is $\omega^{*}$-convergent to 0 . It follows that $u^{*}=\left(I-Q_{m}^{*}\right)\left(z^{*}\right) \in G_{1}$. Finally, it is clear that $\widetilde{\bigcup_{m=1}^{\infty}} H_{m}=\bigcup_{m=1}^{\infty} \tilde{H}_{m}$ and the conclusion $\tilde{\Gamma} \subset G_{1}$ follows. This proves Lemma 1.

The next lemma is a generalization of Lemma 4.1 of [9].

LEMMA 2. Let $Y$ be a Banach space with a shrinking basis $\left\{y_{n}\right\}_{n=1}^{\infty}$ and let $\left\{y_{n}^{*}\right\}_{n=1}^{\infty}$ denote its biorthogonal functionals. Let $E$ be a quotient space of $Y$, let $U: Y \rightarrow E$ be a quotient mapping, and put $e_{n}=U y_{n}, n=1,2, \ldots$ Given $\varepsilon>0$, there is a sequence $\left\{\Psi_{n}\right\}_{n=1}^{\infty} \subset B\left(E^{*}\right)$ satisfying the following two conditions:

(2.1) $\|e\| \leq(1+\varepsilon) \sup _{n}\left|\Psi_{n}(e)\right|$ for every $e \in E$;

(2.2) let $\Delta=$ the $\omega^{*}$-closure of $\left\{\Psi_{n}\right\}_{n=1}^{\infty}$; then there is a sequence $\left\{D_{i}\right\}_{i=1}^{\infty}$ of finite sets of numbers such that for every $\theta \in \Delta$ and $i \geq 1, \theta\left(e_{i}\right) \in D_{i}$.

PROOF. It is easy to see that we can find a biorthogonal system $\left\{x_{n}, \theta_{n}\right\}_{n=1}^{\infty}$ where $\left[x_{n}\right]_{n=1}^{\infty}=E$ and $\theta_{n} \in E^{*}$ such that for every $n \geq 1, e_{n} \in\left[x_{i}\right]_{i=1}^{n}$. Now use Lemma 4.1 of $[9]$ to obtain a sequence $\left\{\Psi_{n}\right\}_{n=1}^{\infty} \subset B\left(E^{*}\right)$ and a sequence $\left\{C_{i}\right\}_{i=1}^{\infty}$ of finite sets of numbers such that for every $\theta \in \Delta=\omega^{*}$-closure of $\left\{\Psi_{n}\right\}_{n=1}^{\infty}$, $\theta\left(x_{i}\right) \in C_{i}$ for $i \geq 1$ and (2.1) holds. Since each $e_{n}=\sum_{i=1}^{n} \theta_{i}\left(e_{n}\right) x_{i}$, it follows that $\theta\left(e_{n}\right)=\sum_{i=1}^{n} \theta_{i}\left(e_{n}\right) \theta\left(x_{i}\right)$. Let $D_{n}=\left\{\sum_{i=1}^{n} \theta_{i}\left(e_{n}\right) c_{i}: c_{i} \in C_{i}, 1 \leq i \leq n\right\}$ then $\left\{D_{n}\right\}_{n=1}^{\infty}$ are the desired sets.

LEMMA 3. Let $E$ be a Banach space with a separable dual space $E^{*}$. Then there is a $\omega^{*}$-compact subset $\Delta$ of $B\left(E^{*}\right)$ such that the following conditions are satisfied:

(2.3) for every $e \in E \sup \{\varphi(e): \varphi \in \Delta\} \leq\|e\| \leq 2 \sup \{\varphi(e): \varphi \in \Delta\}$; 
(2.4) under the $\omega^{*}$-topology, $\Delta$ has a Cantor partition $\left\{\Delta\left(a_{1}, a_{2}, \ldots, a_{n}\right): n \geq 1\right.$, $\left.a_{i} \in D_{i}, 1 \leq i \leq n\right\}$ with a tree $\left\{e^{*}\left(a_{1}, a_{2}, \ldots, a_{n}\right) \in \Delta: e^{*}\left(a_{1}, a_{2}, \ldots, a_{n}\right) \in\right.$ $\left.\Delta\left(a_{1}, a_{2}, \ldots, a_{n}\right), n \geq 1, a_{i} \in D_{i}, 1 \leq i \leq n\right\}$ such that, whenever $e^{*} \in \Delta$ and $\left\{e^{*}\right\}=\bigcap_{n=1}^{\infty} \Delta\left(a_{1}, a_{2}, \ldots, a_{n}\right)$ (for a particular choice of $\left\{a_{i}\right\}_{i=1}^{\infty}$ with $\left.a_{i} \in D_{i}\right)$, then $\lim _{n \rightarrow \infty}\left\|e^{*}-e^{*}\left(a_{1}, a_{2}, \ldots, a_{n}\right)\right\|=0$.

ProOF. By Corollary 8 of $[\mathbf{1}] E$ is a quotient space of a space $Y$ with a shrinking basis $\left\{y_{n}\right\}_{n=1}^{\infty}$. Let $U: Y \rightarrow E$ be a quotient mapping, let $e_{n}=U y_{n}$ and use Lemma 2 to construct the sequence $\left\{\Psi_{n}\right\}_{n=1}^{\infty} \subset B\left(E^{*}\right)$ and the $\omega^{*}$-compact set $\Delta$. Clearly, for every $\theta \in \Delta,\left(U^{*} \theta\right)\left(y_{i}\right) \in D_{i}$. Consider the subset $G$ of $B\left(Y^{*}\right)$ defined by $G=\left\{y^{*} \in B\left(Y^{*}\right): y^{*}\left(y_{i}\right) \in D_{i}\right.$ for all $\left.i \geq 1\right\}$. Clearly $G$ is a $\omega^{*}$-compact subset of $B\left(Y^{*}\right)$ and if $A\left(a_{1}, a_{2}, \ldots, a_{n}\right)=\left\{y^{*} \in G: y^{*}\left(y_{i}\right)=a_{i}, 1 \leq i \leq n\right\}$ where $a_{i} \in D_{i}$ for $1 \leq i \leq n$, then $A\left(a_{1}, a_{2}, \ldots, a_{n}\right)$ is a clopen subset of $G$ in the relative $\omega^{*}$-topology of $Y^{*}$. We have that $A\left(a_{1}, a_{2}, \ldots, a_{n}, a\right) \cap A\left(a_{1}, a_{2}, \ldots, a_{n}, b\right)=\varnothing$ if $a \neq b, a, b \in D_{n+1}$ and $A\left(a_{1}, \ldots, a_{n}\right)=\bigcup_{a \in D_{n+1}} A\left(a_{1}, a_{2}, \ldots, a_{n}, a\right)$. The isome$\operatorname{try} U^{*}: E^{*} \rightarrow Y^{*}$ is both $\omega^{*}$ and norm continuous and it maps $B\left(E^{*}\right)$ into $B\left(Y^{*}\right)$. Hence the set $\Delta=U^{*-1}(G)$ has a Cantor partition $\left\{\Delta\left(a_{1}, a_{2}, \ldots, a_{n}\right): n \geq 1\right.$, $\left.a_{i} \in D_{i}, 1 \leq i \leq n\right\}$ where $\Delta\left(a_{1}, a_{2}, \ldots, a_{n}\right)=U^{*-1}\left(A\left(a_{1}, \ldots, a_{n}\right)\right.$. An element $e^{*}$ of $\Delta$ belongs to $\Delta\left(a_{1}, a_{2}, \ldots, a_{n}\right)$ if $e^{*}\left(e_{i}\right)=a_{i}, 1 \leq i \leq n$; hence, $U^{*} e^{*}=\sum_{i=1}^{\infty} b_{i} y_{i}^{*}$ where $b_{i}=a_{i}$ for $1 \leq i \leq n$. $\left\{e^{*}\right\} \in \bigcap_{n=1}^{\infty} \Delta\left(a_{1}, a_{2}, \ldots, a_{n}\right)$ if and only if $U^{*} e^{*}=\sum_{i=1}^{\infty} a_{i} y_{i}^{*}$. It follows that $\bigcap_{n=1}^{\infty} \Delta\left(a_{1}, a_{2}, \ldots, a_{n}\right)$ is either empty or a one-point set as required. Let us now select the branch points $e^{*}\left(a_{1}, a_{2}, \ldots, a_{n}\right)$. If $n=1, a_{1} \in D_{1}$ and $\Delta\left(a_{1}\right) \neq \varnothing$ let $e^{*}\left(a_{1}\right)$ be an element $e_{0}^{*}$ of $\Delta\left(a_{1}\right)$ such that $\left\|\sum_{i=2}^{\infty}\left(U^{*} e_{0}^{*}\right)\left(y_{i}\right) y_{i}^{*}\right\|$ is minimal. Suppose that for every $1 \leq k \leq n$ and $a_{i} \in D_{i}, 1 \leq i \leq k, e^{*}\left(a_{1}, a_{2}, \ldots, a_{k}\right)$ have been determined and proceed by induction as follows: Let $a_{n+1} \in D_{n+1}$ and assume that $\Delta\left(a_{1}, a_{2}, \ldots, a_{n+1}\right) \neq \varnothing$; if $e^{*}\left(a_{1}, a_{2}, \ldots, a_{n}\right) \in \Delta\left(a_{1}, a_{2}, \ldots, a_{n+1}\right)$, then we put $e^{*}\left(a_{1}, a_{2}, \ldots, a_{n}, a_{n+1}\right)=e^{*}\left(a_{1}, a_{2}, \ldots, a_{n}\right)$. Otherwise let $e^{*}\left(a_{1}, a_{2}, \ldots, a_{n}, a_{n+1}\right)$ be an element $e_{0}^{*}$ of $\Delta\left(a_{1}, a_{2}, \ldots, a_{n}, a_{n+1}\right)$ such that $\left\|\sum_{i=n+2}^{\infty}\left(U^{*} e_{0}^{*}\right)\left(y_{i}\right) y_{i}^{*}\right\|$ is minimal. To prove that the collection $\left\{e^{*}\left(a_{1}, a_{2}, \ldots, a_{n}\right): n \geq 1, a_{i} \in D_{i}, 1 \leq i \leq\right.$ $n\}$ is the desired tree, let $e^{*} \in \Delta$ and assume that $\left\{e^{*}\right\}=\bigcap_{n=1}^{\infty} \Delta\left(a_{1}, a_{2}, \ldots, a_{n}\right)$. There are two cases to consider: if $e^{*}=e^{*}\left(a_{1}, a_{2}, \ldots, a_{k}\right)$ is itself a branch point then, by definition, for each $i>k, e^{*}\left(a_{1}, a_{2}, \ldots, a_{i}\right)=e^{*}\left(a_{1}, a_{2}, \ldots, a_{k}\right)$ and so $\left\|e^{*}-e^{*}\left(a_{1}, a_{2}, \ldots, a_{i}\right)\right\|=0$ for all $i \geq k$. If, on the other hand, $e^{*}$ is not a branch point, then in the sequence $\left\{e^{*}\left(a_{1}, a_{2}, \ldots, a_{i}\right)\right\}_{i=1}^{\infty}$ each element may be repeated only finitely many times. Hence there is a subsequence $\{i(k)\}_{k=1}^{\infty}$ of integers such that the elements $u_{k}^{*}=e^{*}\left(a_{1}, a_{2}, \ldots, a_{i(k)}\right)$ satisfy the condition

$$
\begin{aligned}
& \left\|\sum_{n=i(k)+1}^{\infty}\left(U^{*} u_{k}^{*}\right)\left(y_{n}\right) y_{n}^{*}\right\| \\
& \quad=\min \left\{\left\|\sum_{n=i(k)+1}^{\infty}\left(U^{*} u^{*}\right)\left(y_{n}\right) y_{n}^{*}\right\|: u^{*} \in \Delta\left(a_{1}, a_{2}, \ldots, a_{i(k)}\right)\right\}
\end{aligned}
$$

and each member $e^{*}\left(a_{1}, a_{2}, \ldots, a_{n}\right)$ of the above sequence is equal to an element $e^{*}\left(a_{1}, a_{2}, \ldots, a_{i(k)}\right)$ for some $k \geq 1$. It will, therefore, be sufficient to show that $\lim _{k}\left\|e^{*}-e^{*}\left(a_{1}, a_{2}, \ldots, a_{i(k)}\right)\right\|=0$. To prove this, note $e^{*} \in \Delta\left(a_{1}, a_{2}, \ldots, a_{i(k)}\right)$ 
for each $k$ and hence

$$
\begin{aligned}
\left\|\sum_{n=i(k)+1}^{\infty}\left(U^{*} e^{*}\right)\left(y_{n}\right) y_{n}^{*}\right\| & =\left\|\sum_{n=i(k)+1}^{\infty} a_{n} y_{n}^{*}\right\| \geq\left\|\sum_{n=i(k)+1}^{\infty}\left(U^{*} u_{k}^{*}\right)\left(y_{n}\right) y_{n}^{*}\right\| \\
& =\left\|U^{*} e^{*}\left(a_{1}, a_{2}, \ldots, a_{i(k)}\right)-\sum_{n=1}^{i(k)} a_{n} y_{n}^{*}\right\| .
\end{aligned}
$$

It follows that

$$
\begin{aligned}
\left\|e^{*}-e^{*}\left(a_{1}, a_{2}, \ldots, a_{i(k)}\right)\right\| & =\left\|U^{*} e^{*}-U^{*} e^{*}\left(a_{1}, a_{2}, \ldots, a_{i(k)}\right)\right\| \\
& \leq\left\|\sum_{n=i(k)+1}^{\infty} a_{n} y_{n}^{*}\right\|+\left\|U^{*} e^{*}\left(a_{1}, a_{2}, \ldots, a_{i(k)}\right)-\sum_{n=1}^{i(k)} a_{n} y_{n}^{*}\right\| \\
& \leq 2\left\|\sum_{n=i(k)+1}^{\infty} a_{n} y_{n}^{*}\right\| \underset{k \rightarrow \infty}{\longrightarrow} 0 .
\end{aligned}
$$

This proves Lemma 3.

3. Proof of the Theorem. We first use Lemma 3 to obtain the subset $\Delta$ of $B\left(E^{*}\right)$ such that (2.3) and (2.4) are satisfied. Then we embed $E$ into $C(\Delta)$ using the isomorphic map $V$ defined by $(V e)\left(e^{*}\right)=e^{*}(e)$. It follows that $\frac{1}{2}\|e\| \leq\|V e\| \leq\|e\|$ for all $e \in E$ and the mapping $f: \Delta \rightarrow C(\Delta)^{*}$ defined by $\left(f\left(e^{*}\right)\right)(x)=x\left(e^{*}\right)$ for every $e^{*} \in \Delta$ and $x \in C(\Delta)$ is $\omega^{*}-\omega^{*}$ continuous, i.e., if $u_{n}^{*}, u^{*} \in \Delta$ and $\omega^{*} \lim u_{n}^{*}=$ $u^{*}$, then $\lim _{n} x\left(u_{n}^{*}\right)=x\left(u^{*}\right)$ for every $x \in C(\Delta)$ and so $\omega^{*} \lim f\left(u_{n}^{*}\right)=f\left(u^{*}\right)$. For every subset $\Delta\left(a_{1}, a_{2}, \ldots, a_{n}\right)$, the characteristic function $x\left(a_{1}, a_{2}, \ldots, a_{n}\right)=$ $\chi \Delta\left(a_{1}, a_{2}, \ldots, a_{n}\right)$ is a continuous function on $\Delta$ because $\Delta\left(a_{1}, a_{2}, \ldots, a_{n}\right)$ is both open and closed (in the $\omega^{*}$-topology of $E^{*}$ ). Define for every $n \geq 1$ the projection $P_{n}$ on $C(\Delta)$ by

$$
P_{n} x=\sum_{i=1}^{n} \sum_{a_{i} \in D_{i}} x\left(e^{*}\left(a_{1}, a_{2}, \ldots, a_{n}\right)\right) x\left(a_{1}, a_{2}, \ldots, a_{n}\right)
$$

Clearly $\left\|P_{n}\right\|=1$ and $P_{n} x \rightarrow x$ for every $x \in C(\Delta)$ in norm. Moreover, if $e^{*} \in \Delta$ with $\left\{e^{*}\right\}=\bigcap_{n=1}^{\infty} \Delta\left(b_{1}, b_{2}, \ldots, b_{n}\right), b_{i} \in D_{i}$, and $f\left(e^{*}\right)$ denotes, as above, the corresponding point evaluation functional on $C(\Delta)$, then

$$
P_{n}^{*} f\left(e^{*}\right)=f\left(e^{*}\left(b_{1}, b_{2}, \ldots, b_{n}\right)\right), \quad n=1,2, \ldots
$$

The last part of $(2.4)$ states that $\lim _{n \rightarrow \infty}\left\|e^{*}-e^{*}\left(b_{1}, b_{2}, \ldots, b_{n}\right)\right\|=0$ and, hence, we have

$$
\lim _{n \rightarrow \infty} \sup _{V e \in B(V(E))}\left|\left(f\left(e^{*}\right)-P_{n}^{*} f\left(e^{*}\right)\right)(V e)\right|=0 .
$$


Our construction ensures that $V(E) \cap\left(\bigcup_{n=1}^{\infty} P_{n}(C(\Delta))\right)$ is norm dense in $V(E)$. Indeed, the sequence $\left\{e_{n}\right\}_{n=1}^{\infty}$ spans $E$ and for each $n$ and $k \geq n$ we have that

$$
\begin{aligned}
P_{k} V e_{n} & =\sum_{i=1}^{k} \sum_{a_{i} \in D_{i}}(V e)\left(e^{*}\left(a_{1}, a_{2}, \ldots, a_{k}\right)\right) x\left(a_{1}, a_{2}, \ldots, a_{k}\right) \\
& =\sum_{a_{n} \in D_{n}} \sum_{i=1, i \neq n}^{k} \sum_{a_{i} \in D_{i}} e^{*}\left(a_{1}, a_{2}, \ldots, a_{k}\right)\left(e_{n}\right) x\left(a_{1}, a_{2}, \ldots, a_{k}\right) \\
& =\sum_{a_{n} \in D_{n}} \sum_{i=1, i \neq n}^{k} \sum_{a_{i} \in D_{i}} a_{n} x\left(a_{1}, a_{2}, \ldots, a_{k}\right) .
\end{aligned}
$$

But since

$$
x\left(a_{1}, a_{2}, \ldots, a_{n}\right)=\sum_{i=n+1}^{k} \sum_{a_{i} \in D_{i}} x\left(a_{1}, a_{2}, \ldots, a_{n}, a_{n+1}, \ldots, a_{k}\right),
$$

we get that

$$
\begin{aligned}
P_{k} V e_{n} & =\sum_{a_{n} \in D_{n}} a_{n} \sum_{i=1}^{n-1} \sum_{a_{i} \in D_{i}} x\left(a_{1}, a_{2}, \ldots, a_{n}\right) \\
& =\sum_{i=1}^{n} \sum_{a_{i} \in D_{i}} a_{n} x\left(a_{1}, a_{2}, \ldots, a_{n}\right)=P_{n} V e_{n} .
\end{aligned}
$$

It follows from (2.3) that $V e_{n}=\lim _{k} P_{k} V e_{n}=P_{n} V e_{n}$. Let $E_{0}=$ the linear span of $\left\{e_{n}\right\}_{n=1}^{\infty}$ in $E$ and let $Z_{0}$ denote the linear span of the set $V\left(E_{0}\right) \cup\left\{P_{k} V e: e \in E_{0}\right.$, $k \geq 1\}$ in $C(\Delta)$. Let $P_{0}=0$ and define a new norm $\||\cdot|||$ on $Z_{0}$ by

$$
\||z|\|=\inf \left\{\sum_{i=0}^{m}\left|\lambda_{i}\right|: z=\sum_{i=0}^{m} \lambda_{i}\left(I-P_{i}\right) V e_{i} \text { where } e_{i} \in E_{0} \text { and }\left\|V e_{i}\right\| \leq 1\right\} .
$$

Let $e \in E_{0}$; then obviously $\|V e\|\|\leq V e\|$. On the other hand, for every $z \in Z_{0}$, since $\left\|I-P_{i}\right\| \leq 2$ for all $i$, whenever $z=\sum_{i=0}^{m} \lambda_{i}\left(I-P_{i}\right) V e_{i}$ with $\left\|V e_{i}\right\| \leq 1$ we get that $\|z\| \leq 2 \sum_{i=0}^{m}\left|\lambda_{i}\right|$ and therefore $\|z \mid\| \geq \frac{1}{2}\|z\|$. It follows that if $Z$ is the completion of $Z_{0}$ under $\||| \cdot||$ then the closure of $V E_{0}$ in $Z$ is 4-isomorphic to $E$. Let us now compute the new norms of the projections $\left\{P_{n}\right\}$ (restricted to $Z_{0}$ ). First note that $\left\||| I-P_{n} \mid\right\|=\sup \left\{||\left|\left(I-P_{n}\right)(z)\right| \|:|||z|||=1\right\}$ and that if $\||z|\|=1$ and $\varepsilon>0$ then there exist numbers $\left\{\lambda_{i}\right\}_{i=0}^{m}$ and elements $\left\{V e_{i}\right\}_{i=0}^{m} \subset B\left(V\left(E_{0}\right)\right)$, such that $z=\sum_{i=0}^{m} \lambda_{i}\left(I-P_{i}\right) V e_{i}$ and $\sum_{i=0}^{m}\left|\lambda_{i}\right|<(1+\varepsilon)|\|z \mid\|=1+\varepsilon$. It follows that

$$
\left(I-P_{n}\right) z=\sum_{i=0}^{n} \lambda_{i}\left(I-P_{n}\right) V e_{i}+\sum_{i=n+1}^{m} \lambda_{i}\left(I-P_{i}\right) V e_{i}
$$

Hence $\left\|\left(I-P_{n}\right)(z)\right\| \mid \leq 1+\varepsilon$. It follows that $\left\|\mid\left(I-P_{n}\right)\right\| \|=1$ and therefore $\left\|\left|P_{n} \|\right| \leq 2\right.$ for all $n \geq 1$. Now consider the mapping $g: C^{*}(\Delta) \rightarrow Z_{0}^{*}$ defined for $x^{*} \in C(\Delta)$ by $g\left(x^{*}\right)(z)=x^{*}(z)$ for all $z \in Z_{0}$. We claim that $g$ is a $\omega^{*}-\omega^{*}$ continuous function. Indeed, for every $z \in Z_{0},\||| z \mid\| \geq \frac{1}{2}\|z\|$ hence $\left\|\left|g\left(x^{*}\right)\right|\right\| \leq$ $2\left\|x^{*}\right\|$ and if $x^{*}=\omega^{*} \lim _{n} x_{n}^{*}$ in $C^{*}(\Delta)$ then $x^{*}(z)=\lim _{n} x_{n}^{*}(z)$ and $\sup \left\|x_{n}^{*}\right\|<\infty$. 
It follows that $\sup \left\|\left|g\left(x_{n}^{*}\right)\right|\right\|<\infty, \lim _{n} g\left(x_{n}^{*}\right)(z)=g\left(x^{*}\right)(z)$ for all $z \in Z_{0}$ and so $\omega^{*}-\lim _{n} g\left(x_{n}^{*}\right)=g\left(x^{*}\right)$. In particular, the set $\Omega_{0}=g(f(\Delta))$ is a $\omega^{*}$-compact subset of $Z^{*}$ and, by (2.3), we have for every $e \in E_{0}$

$$
4^{-1}\|e\| \leq\|V e\|\|\leq\| e \| \text {. }
$$

Finally, we claim that $\Omega_{0} \subset\left[P_{n}^{*}\left(z_{0}^{*}\right)\right]_{n=1}^{\infty}$ in $Z_{0}^{*}$. Indeed, it follows from (3.4) that for every $z^{*} \in Z_{0}^{*}$

$$
\left.||\left|z^{*}\right|||=\sup \left\{\mid\left(I-P_{n}^{*}\right) z^{*}\right)(V e) \mid: n>1, V e \in B\left(V\left(E_{0}\right)\right)\right\} .
$$

It follows that if $\omega \in \Omega_{0}$ and $\left.\omega=g(f)\left(e^{*}\right)\right)$ for some $e^{*} \in \Delta$, where $\left\{e^{*}\right\}=$ $\bigcap_{n=1}^{\infty} \Delta\left(a_{1}, a_{2}, \ldots, a_{n}\right)$, then for every $k \geq 1$, by (3.1) and (3.2),

$$
\begin{aligned}
\left\|\mid \omega-P_{k}^{*} \omega\right\| \| & =\sup \left\{\left|\left(\omega-P_{n}^{*} \omega\right)(V e)\right|: n \geq k, V e \in B\left(V\left(E_{0}\right)\right)\right\} \\
& =\sup \left\{\left|\left(f\left(e^{*}\right)-P_{n}^{*} f\left(e^{*}\right)\right) V(e)\right|: n \geq k, V e \in B\left(V\left(E_{0}\right)\right)\right\} \rightarrow 0
\end{aligned}
$$

as $k \rightarrow \infty$. This proves our claim. The space $Z$, having an f.d.d., can be embedded into a space $X$ with a basis $\left\{x_{n}\right\}_{n=1}^{\infty}$ by [8] or [4]. Moreover, the embedding is done in such a way that there is a projection $P$ of $X$ onto $Z$. In addition, it is easy to see that if $\left\{x_{n}^{*}\right\}_{n=1}^{\infty}$ are the basis' biorthogonal functionals, then $P^{*}\left(P_{n}^{*} Z^{*}\right) \subset\left[x_{n}^{*}\right]_{n=1}^{\infty}$ for every $n$. It follows that the set $\Omega=P^{*}\left(\Omega_{0}\right)$ satisfies the conditions of Lemma 1 and therefore there is a space $X_{1}$ with a basis $\left\{\tilde{x}_{n}\right\}_{n=1}^{\infty}$ containing an isomorphic copy of $E$ and there is a $\omega^{*}$-compact subset $\tilde{\Gamma} \subset B\left(\left[\tilde{x}_{n}^{*}\right]_{n=1}^{\infty}\right)$ which is norming for the whole space $X_{1}$. Let $\tilde{\Gamma}_{c}$ denote the $\omega^{*}$-closed convex hull of $\tilde{\Gamma}$; then, by Theorem 3.3 of $[\mathbf{5}], \tilde{\Gamma}_{c} \subset B\left(\left[\tilde{x}_{n}^{*}\right]_{n=1}^{\infty}\right)$. In order to show that $\left\{\tilde{x}_{n}\right\}_{n=1}^{\infty}$ is a shrinking basis it suffices to prove that $X_{1}^{*}=\left[\tilde{x}_{n}^{*}\right]_{n=1}^{\infty}$. If this is not the case then there is a $z^{*} \in B\left(X_{1}^{*}\right)$ with the norm distance $\left|z^{*}-\left[\tilde{x}_{n}^{*}\right]_{n=1}^{\infty}\right|>\frac{1}{2}$ and, in particular, $\left|z^{*}-\tilde{\Gamma}_{c}\right|>\frac{1}{2}$. The standard separation theorem for the $\omega^{*}$-topology implies the existence of a $z \in X_{1}$ such that $z^{*}(z)>\sup \left\{y^{*}(z): y^{*} \in \tilde{\Gamma}_{c}\right\}$. It follows that $z^{*}(z)>|z|$ contradicting the fact that $\left|z^{*}\right| \leq 1$. This proves the Theorem.

REMARK 2. The use of [8] or [4] in the last part of the proof is quite artificial. In fact, there is a natural basis of $C(\Delta)$ the usual projections $\left\{Q_{n}\right\}_{n=1}^{\infty}$ of which could be used instead of $\left\{P_{n}\right\}_{n=1}^{\infty}$. We would then directly get the desired space with a basis. However the computation of $\left\|\omega-Q_{n}^{*} \omega\right\| \|$ becomes complicated while $\left\|\left|\omega-P_{k}^{*} \omega\right|\right\|$ is a simple expression; [8] or [4] is thus used to save us the unnecessary complication.

\section{REFERENCES}

1. W. J. Davis, T. Figiel, W. B. Johnson and A. Pełczyński, Factoring weakly compact operators, J. Funct. Anal. 17 (1974), 311-327.

2. D. W. Dean, J. Singer and L. Sternbach, On shrinking basis sequences in Banach spaces, Studia Math. 40 (1971), 23-33.

3. W. B. Johnson and H. P. Rosenthal, On $\omega^{*}$-basic sequences and their applications to the study of Banach spaces, Studia Math. 43 (1972), 77-92.

4. W. B. Johnson, H. P. Rosenthal and M. Zippin, On bases, finite dimensional decompositions and weaker structures in Banach spaces, Israel J. Math. 9 (1971), 488-506.

5. R. Haydon, An extreme point criterion for separability of a dual Banach space and a new proof of a theorem of Corson, Quart. J. Math. Oxford 27 (1976), 378-385.

6. J. Lindenstrauss and L. Tzafriri, Classical Banach spaces, Vol. I, Springer-Verlag, Berlin, Heidelberg and New York, 1977. 
7. A. Pełczyński, Some problems on bases in Banach and Frechet spaces, Israel J. Math. 2 (1964), 132-138.

8. __ Any separable Banach space with the bounded approximation property is a complemented subspace of a Banach space with a basis, Studia Math. 40 (1971), 239-242.

9. M. Zippin, The separable extension problem, Israel J. Math. 26 (1977), 372-387.

Department of Mathematics, The Hebrew University of Jerusalem, JerusaLEM, ISRAEL 